\title{
Deformation and Recrystallization of Tensile-deformed or Rolled Fe-3\%Si Alloy Single Crystals
}

\author{
Tatsuya OKADA, Masaru SAKAGUCHI, ${ }^{1)}$ Osamu ASHIDA, ${ }^{1)}$ Minoru TAGAMI and Fukuji INOKO \\ Department of Mechanical Engineering, Tokushima University, Tokushima 770-8506 Japan. \\ 1) Graduate Student, Tokushima University, Tokushima 770-8506 Japan.
}

(Received on September 2, 2004; accepted in final form on November 4, 2004)

\begin{abstract}
Fe-3\% Si alloy single crystal samples with various tensile directions were deformed to strains of 0.25 to 0.65 and subsequently annealed. Necking occurred and work-hardening was very small in all tensile-deformed samples. After annealing, no recrystallized grain was formed. Fe-3\%Si alloy single crystal sample of $\{111\}\langle 112\rangle$ orientation was lightly rolled to $25 \%$ and subsequently annealed. Many recrystallized grains were formed in the rolled sample. The orientation of recrystallized grains formed at the rolled surface was totally different from the orientation of those formed in the interior of the sample. These results are discussed based on the tendency for cross-slip and the dislocation network model for nucleus of recrystallized grain.
\end{abstract}

KEY WORDS: Fe-3\%Si alloy; single crystal; deformation; recrystallization.

\section{Introduction}

Recrystallization has long been studied because it is virtually the only measure for texture control of metals and alloys in which phase transformation does not occur in appropriate temperature range. Because almost all structural metallic materials are polycrystalline and heavy plastic deformation is applied in their production process, the main stream of recrystallization study has been the evaluation of texture changes associated with large deformation and subsequent annealing.

For the complete understanding of recrystallization, we should examine the dislocation mechanisms of recrystallization through detailed analysis of deformation microstructures, e.g. slip morphology and associated local orientation change, because recrystallized grains (RGs) are formed through migration, rearrangement and annihilation of dislocations accumulated in deformed materials. Experimental studies using single crystals and bicrystals as samples are important for unambiguous evaluation of the deformation microstructure. ${ }^{1-9)}$ In addition, relatively small strain is preferred for slip analysis of such samples.

Inoko and coworkers ${ }^{5-9}$ ) have been studying the deformation microstructures of tensile-deformed aluminum single crystals and bicrystals, and their initial stage of recrystallization. Recently, they reported interesting results of $\langle 001\rangle$, $\langle 112\rangle$ and $\langle 111\rangle$ oriented aluminum single crystals tensiledeformed at room temperature, which exhibited multiple slip from the initial stage of plastic deformation. ${ }^{10-14)}$ For these single crystals, bands of large orientation gradient such as deformation bands were not formed. Hence, the misorientation in their deformed microstructures was within a range of $5^{\circ}$. Despite such macroscopically uniform deformation, RGs were formed in the $\langle 111\rangle$ and $\langle 112\rangle$ single crystals after annealing. ${ }^{10,11)}$ Many RGs had $\langle 111\rangle$ rotation relationship with the orientation of the deformation matrix. On the other hand, recrystallization did not occur in the $\langle 001\rangle$ single crystal. ${ }^{10)}$ This difference reflects well their deformation behaviors, especially tendency to cross-slip. Among these single crystals, the work-hardening rate was the largest for the $\langle 111\rangle$ single crystal and the nominal tensile stresses at a strain of 0.22 were $22 \mathrm{MPa}, 32 \mathrm{MPa}$ and $71 \mathrm{MPa}$ for the $\langle 001\rangle,\langle 112\rangle$ and $\langle 111\rangle$ single crystals, respectively. In the $\langle 001\rangle$ single crystal, cross-slip with large step height of approximately $100 \mu \mathrm{m}$ developed, which resulted in a release of strain accumulation at the intersections of slip bands, most likely recrystallization sites for single crystals of multiple slip orientation. On the other hand, cross-slip with large step height was suppressed in the $\langle 111\rangle$ and $\langle 112\rangle$ single crystals.

Although extensive studies have been conducted for texture formations associated with deformation and annealing, relatively smaller numbers of studies have been done for dislocation mechanisms of recrystallization in BCC metals. Okada et al. ${ }^{15,16)}$ investigated slip deformation and recrystallization of compressed $\mathrm{Fe}-30 \% \mathrm{Cr}$ alloy single crystals. The RGs formed in a shear band had $\langle 110\rangle$ rotation relationship with the orientation of the shear band. In the proceeding paper, we reported that in a tensile-deformed $\{111\}\langle 112\rangle \mathrm{Fe}-3 \% \mathrm{Si}$ alloy single crystal, recrystallization did not occur even in the neck portion of the sample. ${ }^{17)} \mathrm{On}$ the other hand, in a tensile-deformed $\mathrm{Fe}-3 \% \mathrm{Si}$ alloy bicrystal containing $\{111\}\langle 112\rangle$ and $\{001\}\langle 110\rangle$ grains, slip in the $\{111\}\langle 112\rangle$ grain changed markedly from that in the single crystal due to the constrain from the grain boundary, and RGs were formed only in the non-uniformly deformed region close to the grain boundary in the $\{111\}\langle 112\rangle$ grain.

From the experimental results described above, close re- 
lationship between deformation and recrystallization is expected in BCC metal single crystals. One major objective of the present study is to investigate deformation and annealing behaviors of single crystals of $\mathrm{Fe}-3 \% \mathrm{Si}$ alloy with various tensile orientations. The other objective is to study the recrystallization in uniformly deformed $\mathrm{Fe}-3 \% \mathrm{Si}$ alloy single crystal of $\{111\}\langle 112\rangle$ orientation deformed by light rolling.

\section{Experimental Procedure}

Single crystal samples were spark-cut from a coarsegrained $\mathrm{Fe}-3 \% \mathrm{Si}$ alloy polycrystal prepared by JFE Steel Co. Ltd. The dimension of the gauge portion of the samples for tensile deformation was $4 \mathrm{~mm} \times 12 \mathrm{~mm} \times 2 \mathrm{~mm}$ in width, length and thickness. Four samples were prepared for tensile deformation. The tensile directions of the samples are plotted in a stereographic triangle in Fig. 1. Sample No. 4 had the same $\{111\}\langle 112\rangle$ orientation as the one in the proceeding paper. ${ }^{17)}$ These samples were tensile-deformed at the strain rate of $5.6 \times 10^{-5}$ to $1.4 \times 10^{-4} \mathrm{~s}^{-1}$, to strains of 0.25 to 0.65 . The dimension of the sample for rolling was $4 \mathrm{~mm} \times 46 \mathrm{~mm} \times 2 \mathrm{~mm}$ in width, length and thickness. The initial orientation of the rolled sample is presented in Fig. 2. The normal direction (ND) of the rolled surface and the rolled direction $(\mathrm{RD})$ were [111] and [21̄1] , respectively. In order to obtain uniform deformation, relatively light rolling of $25 \%$ was applied. The deformed microstructure was examined using a scanning electron microscope (SEM JEOL JSM-6400). Orientation change associated with deformation was determined from electron channeling patterns

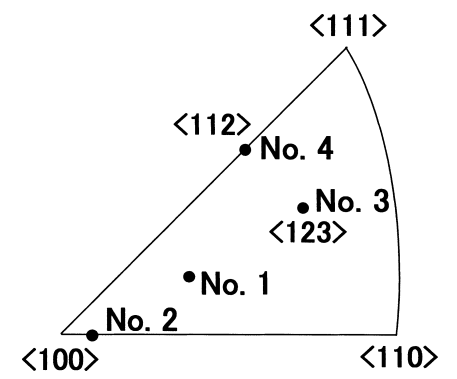

Fig. 1. Tensile directions of single crystal samples plotted in a stereographic triangle. The tensile directions of the sample No. 3 and No. 4 are $\langle 123\rangle$ and $\langle 112\rangle$, respectively.

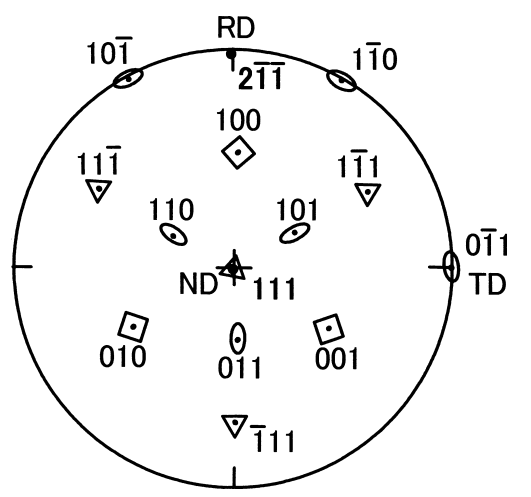

Fig. 2. The initial orientation of the rolled sample. The rolling direction (RD) and normal direction (ND) are [2 $\overline{1} \overline{1}]$ and [111], respectively. The tangential direction (TD) is [011].
(ECPs) taken with the SEM.

The annealing was conducted for the tensile-deformed samples at 1173 to $1273 \mathrm{~K}$ for 180 to $780 \mathrm{~s}$. The rolled sample was annealed at $873 \mathrm{~K}$ for $720 \mathrm{~s}$. After annealing, orientation of RGs was determined from their ECPs. For the rolled sample, the rolled surface was removed by 0.25 $\mathrm{mm}$ to observe the formation of RGs in the interior of the sample.

\section{Results}

\subsection{Tensile-deformed Samples}

Nominal stress-strain curves for the four tensile-deformed samples are presented in Fig. 3. In all samples, necking started from the early stage of plastic deformation. After the neck formation, plastic deformation proceeded mainly in the neck portion. Hence, nominal work-hardening was very small for all samples. The overall appearance of Sample No. 4 with the nominal strain of 0.25 is shown in Fig. 4. After annealing, the four samples did not recrystallize. The difficulty in recrystallization for tensile-deformed $\mathrm{Fe}-3 \% \mathrm{Si}$ single crystals is discussed in Sec. 4.1.

\subsection{Rolled Sample}

SEM image of the side surface (TD surface) of the rolled sample is presented in Fig. 5. Narrow folds are recognized. The arrangement of the folds is totally different from that of shear bands formed in $60-70 \%$ rolled $\mathrm{Fe}-3 \% \mathrm{Si}$ alloy single crystals of $\{111\}\langle 112\rangle$ orientation. ${ }^{1-4)}$ Since the for-

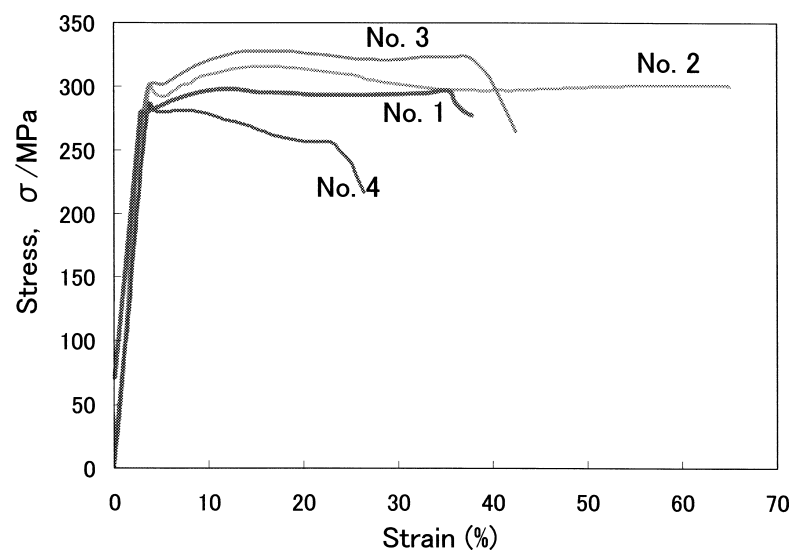

Fig. 3. Nominal stress-strain curves for the tensile-deformed single crystal samples.

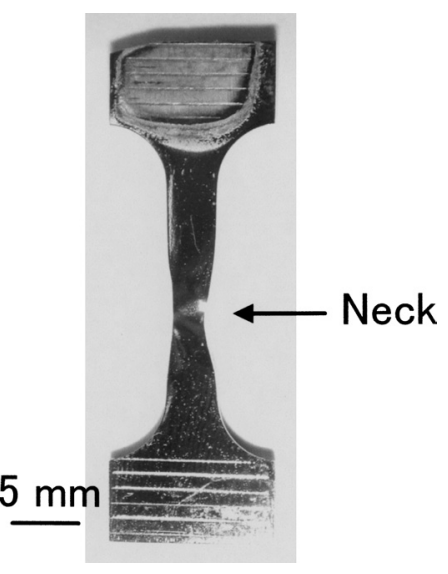

Fig. 4. The shape of the tensile-deformed sample No. 4. 


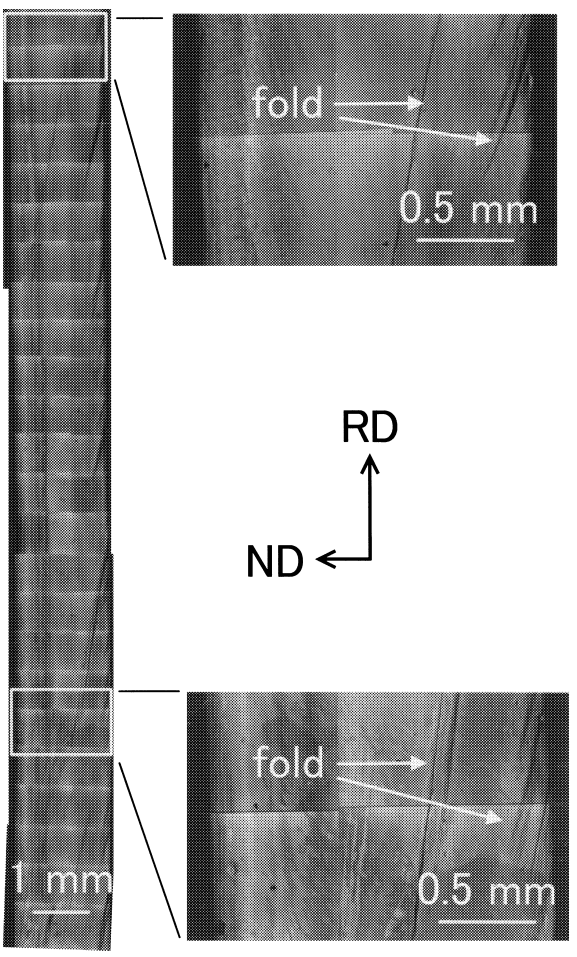

Fig. 5. The TD surface of the rolled sample.

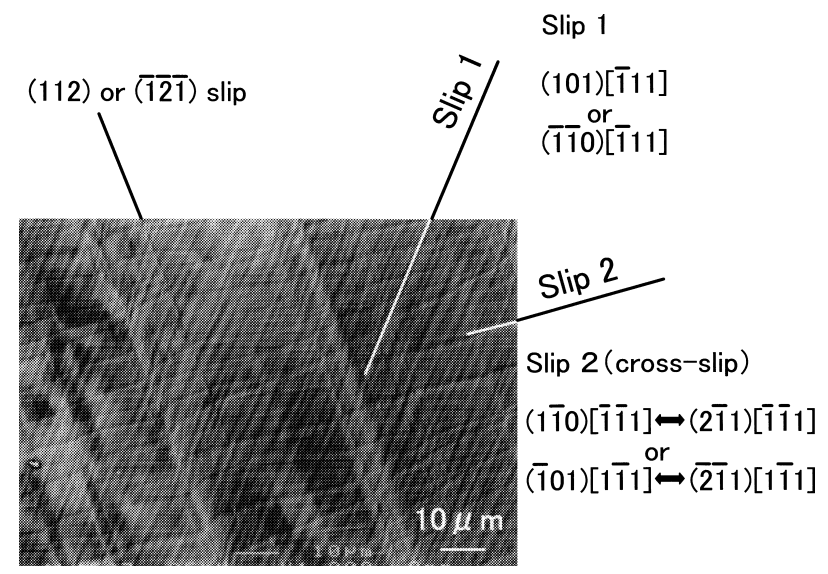

Fig. 6. Typical slip bands in the TD surface of the rolled sample.

mation of folds in the present sample did not affect its recrystallization, we consider that the deformation was almost uniform. A typical SEM image of slip bands is presented in Fig. 6. Slips on $\{110\}$ planes and $\{112\}$ planes were observed. Most slip bands are straight except for Slip

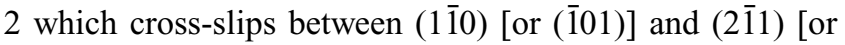
(2̄11)] planes.

After annealing, RGs were formed. Recrystallization observed in the TD surface is presented in Fig. 7. Since the arrangement of RGs had no relationship with the position of the folds, we consider that recrystallization in the present sample occurred in uniformly deformed sample. The optical microscopic image of the ND surface along with its schematic is presented in Fig. 8. The orientation of 79 RGs was measured and compared with that of non-recrystallized region, i.e. deformed matrix. Out of $79 \mathrm{RGs}, 44 \mathrm{RGs}$ had $\langle 110\rangle$ rotation relationship with the deformed matrix. The rotation axes are classified in Table $\mathbf{1}$. In the present study, $\langle 112\rangle$ rotation relationship was not checked because it is

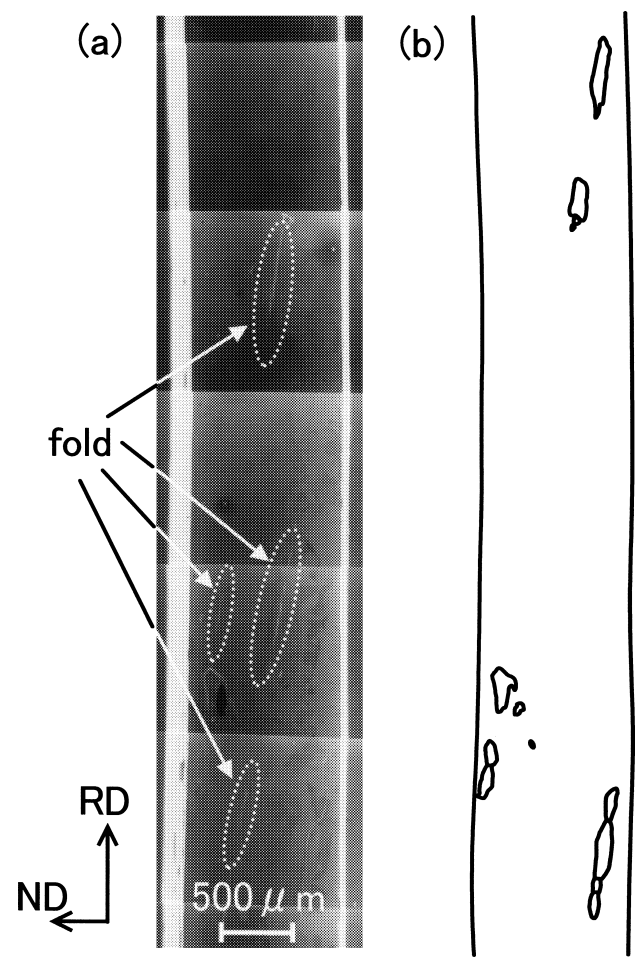

Fig. 7. (a) SEM image of the TD surface of the rolled sample after annealing. Positions of some of the folds are indicated by elliptic marks. (b) A schematic of (a). Grain boundaries around recrystallized grains are drawn.
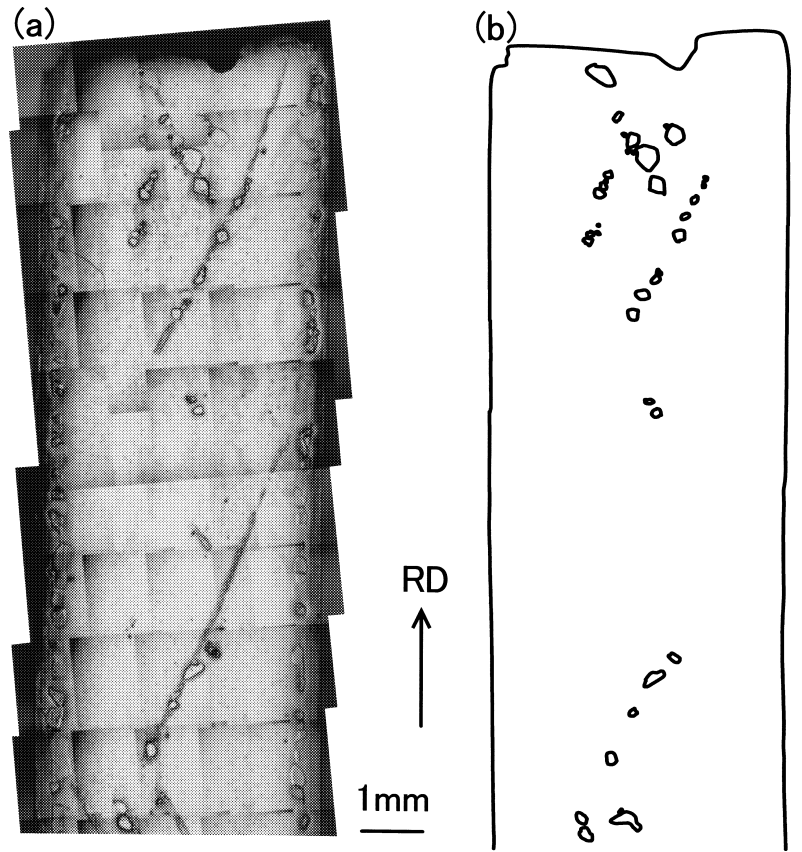

Fig. 8. (a) Optical micrograph of the ND surface of the rolled sample after annealing. (b) A schematic of (a).

generally found in RGs formed at shear bands in heavily (60 to $70 \%$ ) rolled $\mathrm{Fe}-3 \% \mathrm{Si}$ alloy single crystals. About the half of $\langle 110\rangle$-rotated RGs (20 out of 44) were rotated about the TD ([011]) axis. The $\langle 110\rangle$ pole figure of such RGs is presented in Fig. 9. (In the figure, the orientation of the deformed matrix was determined by averaging the orientation of several points in non-recrystallized region. The misorientation of the deformed matrix was within a range of $5^{\circ}$.) 
Table 1. Rotation axis of RGs formed at the rolled surface with respect to the matrix.

\begin{tabular}{|c|c|}
\hline$[110]$ & 9 \\
\hline$[101]$ & 6 \\
\hline$[011]$ & 5 \\
\hline$[10 \overline{1}]$ & 2 \\
\hline$[1 \overline{1} 0]$ & 2 \\
\hline$[0 \overline{1} 1]$ & 20 \\
\hline Twin orientation & 0 \\
\hline Others & 35 \\
\hline Total & 79 \\
\hline
\end{tabular}

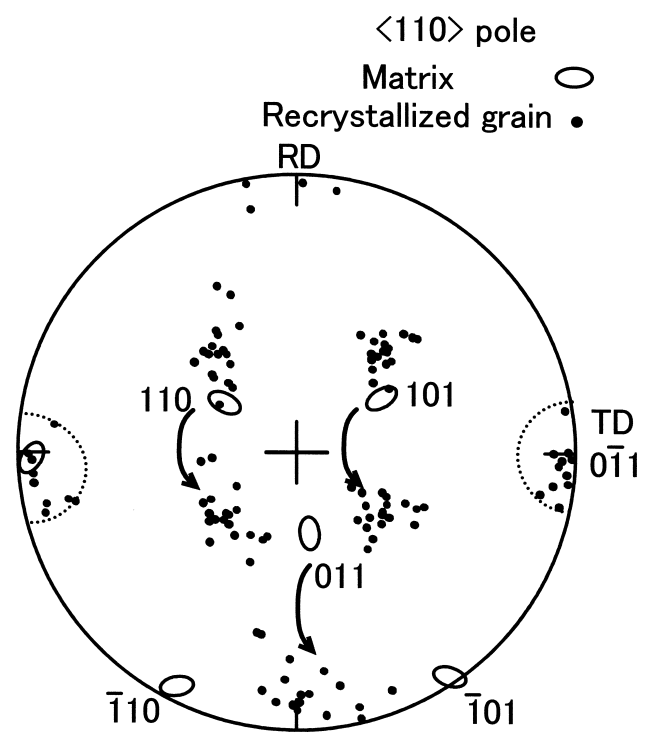

Fig. 9. $\langle 110\rangle$ pole figure of RGs rotated about the TD ([011]) axis with respect to the deformed matrix. The RGs were formed at the rolled surface.

The sense of rotation is singular, that is, the RGs are rotated counterclockwise about the [011] axis.

The rolled surface was removed by $0.25 \mathrm{~mm}$ to observe recrystallization in the interior of the sample where the direct effect of the roller, i.e. friction, is considered to be smaller. The arrangement of RGs is presented in Fig. 10, which is different from that at the rolled surface (Fig. 8). The orientation of 104 recrystallized grains were measured and compared with that of the deformed matrix. The results are summarized in Table 2. The occurrence of each $\langle 110\rangle$ rotation relationship is different from that observed at the rolled surface (Table 1). The major difference is the very small number of occurrences of the TD ([011])-rotated RGs in the interior; $1 / 104$ compared with the high value of 21/79 at the rolled surface. We also note large number of occur-

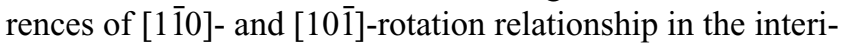
or of the sample, 20/104 and 9/104, respectively. The $\langle 110\rangle$

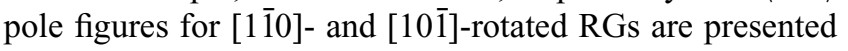
in Figs. 11 and 12, respectively. The interesting feature is

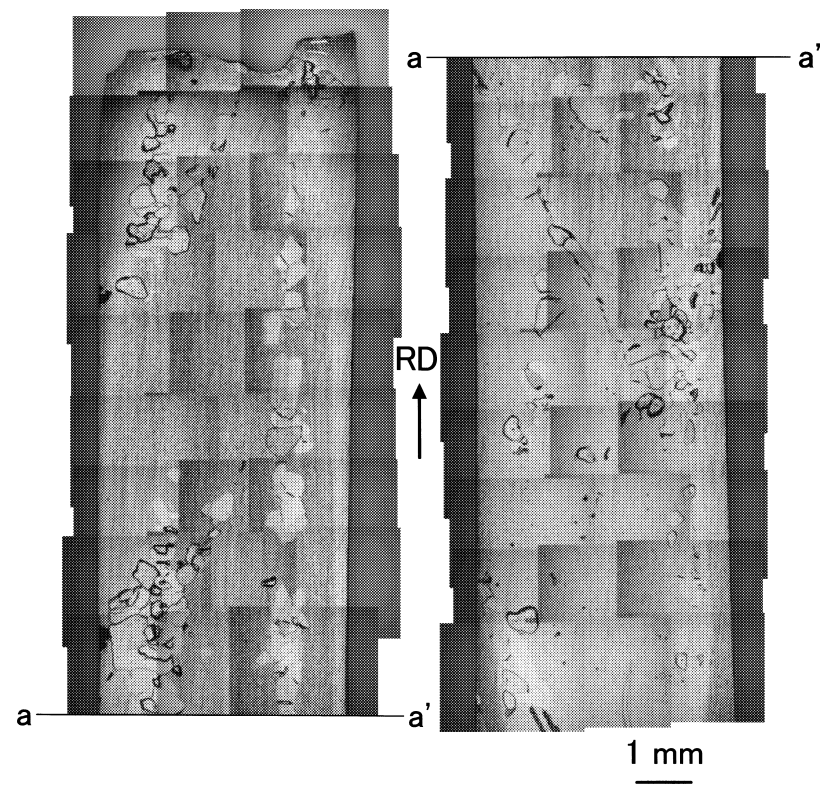

Fig. 10. RGs formed in the interior of the rolled sample.

Table 2. Rotation axis of RGs formed in the interior with respect to the matrix.

\begin{tabular}{|c|c|}
\hline$[110]$ & 9 \\
\hline$[101]$ & 8 \\
\hline$[011]$ & 2 \\
\hline$[10 \overline{1}]$ & 9 \\
\hline$[1 \overline{1} 0]$ & 20 \\
\hline$[0 \overline{1} 1]$ & 1 \\
\hline Twin orientation & 3 \\
\hline Others & 52 \\
\hline Total & 104 \\
\hline
\end{tabular}

that the sense of rotation is almost singular, that is, most RGs are rotated counterclockwise about the [1 10$]$-axis or clockwise about the [10ī]-axis. The singular rotation of RGs was reported in compressed single crystals of Fe$30 \% \mathrm{Cr}$ alloy. ${ }^{15,16)}$ In the compressed $\mathrm{Fe}-30 \% \mathrm{Cr}$ single crystals, a shear band was formed and recrystallization occurred only in the shear band. The counterclockwise rotation of RGs with respect to the orientation of the shear band was opposite to the deformation-induced clockwise rotation of the shear band from the initial orientation. Such singular rotation was explained by dislocation network model of RGs. ${ }^{15,16)}$ Although it is difficult to apply the same discussion to the present case due to the complexity of rolling deformation, the singular rotation of RGs is noteworthy. 


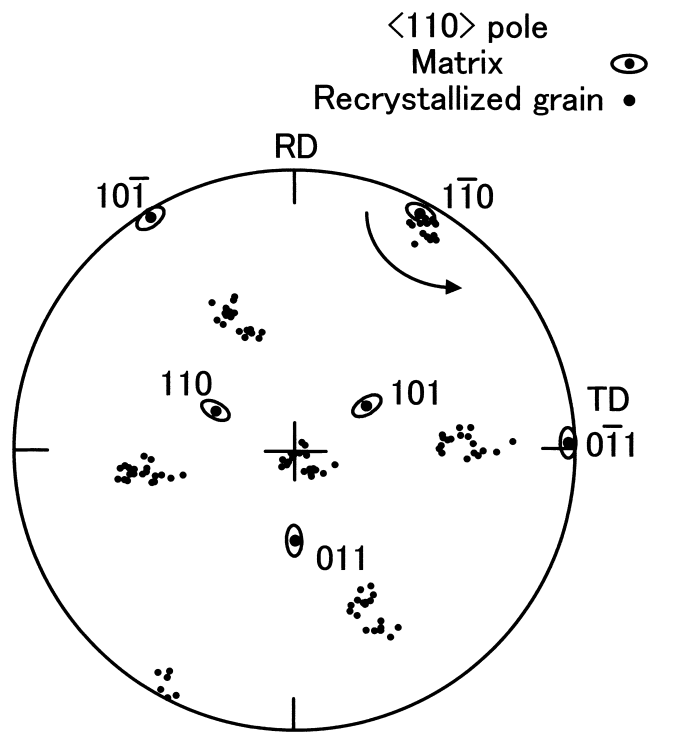

Fig. 11. Orientation of RGs rotated about the [11̄0] axis with respect to the deformed matrix. The RGs were formed in the interior of the sample.

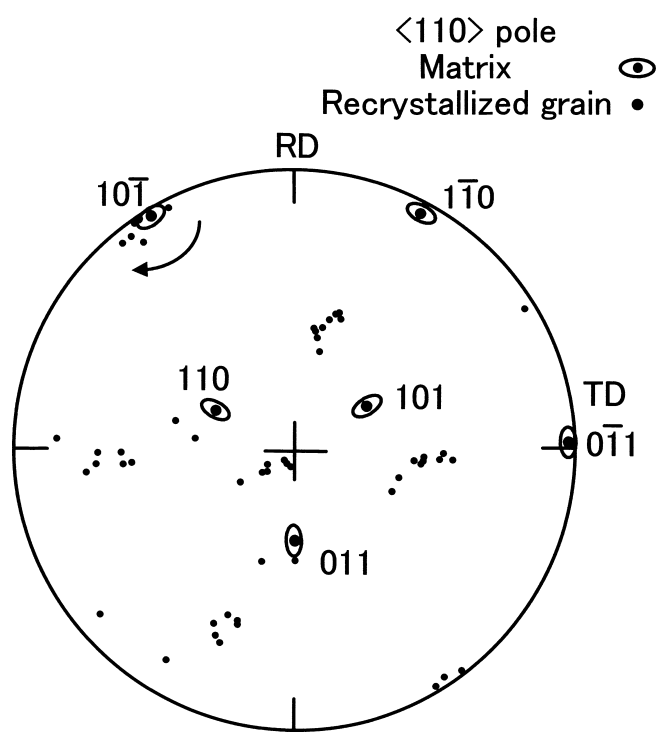

Fig. 12. Orientation of RGs rotated about the [101] axis with respect to the deformed matrix. The RGs were formed in the interior of the sample.

\section{Discussion}

\subsection{Relationship between Deformation and Recrystal- lization in Tensile-deformed Single Crystals}

For tensile-deformed and subsequently annealed single crystals of aluminum, it is known that recrystallization behavior varies with their tensile direction. Inoko and coworkers $^{10-13)}$ have conducted a series of studies for $\langle 001\rangle-,\langle 112\rangle$ and $\langle 111\rangle$-oriented aluminum single crystals. When deformation was applied at room temperature (RT), prominent cross-slip as large as $100 \mu \mathrm{m}$ occurred in the $\langle 001\rangle$ single crystal. The flow stress saturated at the early stage of plastic deformation; the work-hardening was very small. The slip bands at the surface were coarse and the interior dislocation microstructure was characterized by coarse cells bounded by cell walls with low dislocation density. On the contrary, in the $\langle 111\rangle$ single crystal, cross-slip with large step height was suppressed. The slip bands were very fine and the cell size was small, bounded by cell walls with high dislocation density. The work hardening of the $\langle 111\rangle$ single crystals was very large. At a nominal strain of 0.22 , the flow stress of the $\langle 111\rangle$ single crystal (71 MPa) was about three times as large as that of the $\langle 001\rangle$ single crystal (22 MPa). The slip morphology of the $\langle 112\rangle$ single crystal was similar to that of the $\langle 111\rangle$ crystal with relatively low flow stress ( $32 \mathrm{MPa}$ at a strain of 0.22 ). After annealing, very fine RGs were formed in the $\langle 111\rangle$ single crystal. On the other hand, RGs were not formed in the $\langle 001\rangle$ single crystal. In the $\langle 112\rangle$ single crystal, coarse RGs were formed. The number of RGs in the $\langle 112\rangle$ single crystal was smaller than that in the $\langle 111\rangle$ single crystal, which suggests lower density of recrystallization sites in the former.

The close relationship between slip behavior and recrystallization was confirmed in $\langle 001\rangle$ aluminum single crystals deformed in tension at liquid nitrogen temperature (LNT). ${ }^{12,14)}$ The flow stress of the $\langle 001\rangle$ single crystal deformed at LNT (106 MPa at the strain of 0.22 ) is about five times as large as the flow stress obtained at RT (22 MPa). Cross-slip with large-step height was suppressed in the LNT crystal. The slip bands were very fine similar to that of $\langle 111\rangle$ single crystal. After annealing, coarse RGs were formed.

The above results are explained by the dependence of cross-slip on tensile directions for FCC single crystals. It is known that the ratio of the Schmid factor of cross slip system $\left(m_{\mathrm{c}}\right)$ to that of primary slip system $\left(m_{\mathrm{p}}\right)$ depends on the tensile direction. ${ }^{18)}$ For the tensile directions on the line connecting the $\langle 112\rangle$ and $\langle 011\rangle$ poles in a stereographic triangle, the $m_{\mathrm{c}} / m_{\mathrm{p}}$ ratio is zero. For tensile directions staying in the area close to the $\langle 111\rangle$ pole, the $m_{\mathrm{c}} / m_{\mathrm{p}}$ ratio takes negative values, that is, cross-slip is suppressed. For tensile directions in the area close to the $\langle 001\rangle$ pole, the $m_{\mathrm{c}} / m_{\mathrm{p}}$ ratio takes positive values, that is, cross-slip is promoted. The tendency for cross-slip also depends on deformation temperature. When temperature is lowered, cross-slip is suppressed due to lower thermal activation. Here we should note that the above discussion is based only on geometrical consideration. For FCC metals with low stacking fault energy such as copper and nickel, care must be taken for their difficulty in cross-slip even at RT.

For tensile-deformed single crystals of BCC metals such as $\mathrm{Fe}-3 \% \mathrm{Si}$, the $m_{\mathrm{c}} / m_{\mathrm{p}}$ ratio always takes positive values. For tensile direction on the $\langle 112\rangle-\langle 011\rangle$ line, the ratio takes the minimum value of 0.5 . This suggests very easy crossslip for any tensile direction of single crystal of BCC metals. Hence, the strain accumulation is considered to be small at the intersections of slip bands, the most likely positions of recrystallization nucleus in tensile-deformed single crystals of multiple-slip orientation. As a result, the workhardening is very small. Because of small strain accumulation, such crystals are unlikely to recrystallize when annealed.

\subsection{Recrystallization Nucleus}

For uniformly deformed crystals, we should assume nucleation sites for recrystallization other than deformation bands. Inoko and coworkers ${ }^{19,20)}$ analyzed the deformation at the intersections of slip bands and proposed that such in- 


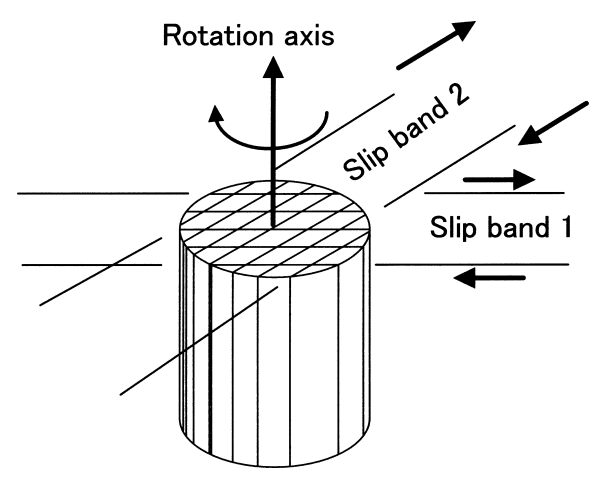

Fig. 13. A model of RG nucleus formed at the intersection of two slip bands. In this figure, shear of two slip bands rotates the nucleus in the clockwise direction.

tersection could be the nucleation sites of RGs. A schematic of their model is presented in Fig. 13. The nucleus of RG is bounded by two sets of dislocation networks introduced by intersecting slips. From geometrical consideration of Fig. 13, it is apparent that the rotation axis of $R G$ is normal to the plane on which two slip directions coexist. Hence, for BCC metals, rotation axis of RG is $\langle 110\rangle$.

In the present study, the predominant rotation axis of RGs formed at the rolled surface is [011] (TD). In the interior of the sample, the predominant rotation axes are [101] and [1 10$]$. Following the geometrical model of Fig. 12, the

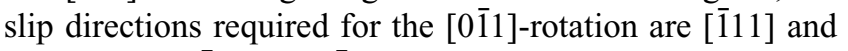
[111]. For [10 $\overline{1}]$ - and [1 10$]$-rotations, the corresponding slip

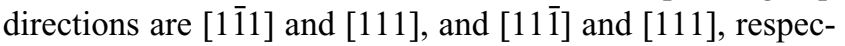
tively. Here it should be noted that for all rotations slip along the [111] direction is involved. Since slip systems with slip direction parallel to [111] (ND) are minor ones in the present sample, their activation is probably limited in small regions. In addition, such slip systems do not produce steps on the TD surface. Hence, it is virtually impossible to observe their slip bands in SEM images such as Fig. 6. In the above mentioned model, we assume a combination of major slip systems such as those with slip direction parallel

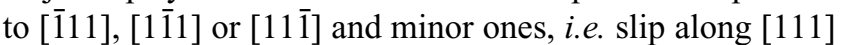
direction for the formation of a RG nucleus. The possibility that such a combination acts as a RG nucleus was proposed for recrystallization in aluminum single crystals tensile-deformed along $\langle 001\rangle$ direction at LNT and subsequently annealed..$^{10,13,14)}$ In such single crystals, in addition to eight primary slip systems, activation of slip systems with Schmid factor of zero was induced by the local strain accumulation caused by the complete suppression of prominent cross-slip. The formation of RGs rotated about four $\langle 111\rangle$ axes in both clockwise and counterclockwise directions with respect to the deformed matrix was geometrically explained by the combination of major and minor slip systems. We consider that similar explanation is applicable to the recrystallization of the present sample. The variation of predominant rotation axis of RGs along the thickness of the sample is attributable to different degree of activation of the slip along the [1111] direction, that is, the slip along the
[111] direction almost parallel to the RD is active at the rolled surface probably due to friction of the roller, but its activity diminishes in the interior of the sample.

\section{Summary}

$\mathrm{Fe}-3 \% \mathrm{Si}$ alloy single crystal samples with various tensile directions were deformed to nominal strains of 0.25 to 0.65 and subsequently annealed. Neck developed in all samples. Recrystallization did not occur even in the neck portion. This is probably due to the fact that in tensile-deformed BCC metal single crystals, cross-slip easily occurs for all tensile directions and strain accumulation is small.

Fe-3\%Si alloy single crystal sample of $\{111\}\langle 112\rangle$ orientation was lightly rolled to $25 \%$. The sample was almost uniformly deformed without shear band formation. After annealing, many RGs were formed. At the ND surface, a large number of RGs were rotated about the TD axis with respect to the deformed matrix orientation. However, the ratio of TD-rotated RGs decreased in the interior of the sample. This is probably due to the difference in slip activity almost parallel to the RD direction, which was caused by the friction from the roller.

\section{REFERENCES}

1) T. Taoka, E. Furubayashi and S. Takeuchi: Trans. Iron Steel Inst. Jpn., 7 (1967), 95.

2) E. Furubayashi: Trans. Iron Steel Inst. Jpn., 9 (1969), 222.

3) E. Furubayashi: ISIJ Int., 38 (1998), 510.

4) T. Toge, M. Muraki, M. Komatsubara and T. Obara: ISIJ Int., 38 (1998), 524.

5) K. Kashihara, M. Tagami, H. Tanaka, T. Okada and F. Inoko: Mater. Trans., 42 (2001), 381.

6) T. Okada, L. Ikeda, X. Huang, J. A. Wert, K. Kashihara and F. Inoko: Mater. Trans., 42 (2001), 1938.

7) K. Kashihara and F. Inoko: Acta Mater., 49 (2001), 3051.

8) J. A. Wert, X. Huang and F. Inoko: Proc. R. Soc. (London), A459 (2003), 85 .

9) T. Okada, X. Huang, K. Kashihara, F. Inoko and J. A. Wert: Acta Mater, 51 (2003), 1827.

10) M. Tagami, K. Kashihara, T. Okada and F. Inoko: J. Jpn. Inst. Met., 64 (2000), 535.

11) M. Tagami, K. Kashihara, T. Okada and F. Inoko: J. Jpn. Inst. Met., 65 (2001), 517

12) M. Tagami, K. Kashihara, T. Okada and F. Inoko: J. Jpn. Inst. Met., 65 (2001), 672

13) M. Tagami, K. Kashihara, T. Okada and F. Inoko: Mater. Trans., 42 (2001), 2013

14) T. Miyazaki, M. Tagami, T. Hama, H. Ogawa, T. Okada and F. Inoko: J. Jpn. Inst. Met., 68 (2004), 342.

15) T. Okada, M. Tagami, K. Kashihara and F. Inoko: ISIJ Int., 38 (1998), 518.

16) T. Okada, W.-Y. Liu and F. Inoko: Mater. Trans. JIM, 40 (1999), 586.

17) T. Okada, M. Sakaguchi, O. Ashida, M. Tagami and F. Inoko: Mater. Trans., 45 (2004), 415.

18) J. Diel, M. Kause, W. Offenhauser and W. Staubwasser: Z. Metallkd., 45 (1954), 489

19) F. Inoko, T. Okada, M. Tagami and K. Kashihara: Proc. 4th Int. Conf. on Recrystallization and Related Phenomena, ed. by T. Sakai and H. G. Suzuki, JIM, Sendai, (1999), 131.

20) F. Inoko, T. Okada, M. Tagami and K. Kashihara: Proc. 21st Risø Inter. Symp., ed. by N. Hansen et al., Risø National Laboratory, Roskilde, (2000), 365. 